\title{
Diseño De Un Modelo De Vivienda Ecológica Con Bambú Para La Zona Rural De Yantzaza Zamora Chinchipe, Ecuador
}

\author{
Oswaldo Malla \\ Master en Planificación y Ordenamiento Territorial para el Desarrollo \\ Carlos Hidalgo \\ Magister en Diseño Urbano y Territorial \\ Rita Vásconez \\ Magister en Turismo Sostenible y Desarrollo Local \\ Escuela Superior Politécnica del Chimborazo \\ - Extensión Norte Amazónica, Ecuador
}

URL:http://dx.doi.org/10.19044/esj.2019.v15n11p99

\section{Resumen}

El estudio se desarrolló en el cantón Yantzaza, perteneciente a la provincia de Zamora Chinchipe. Este trabajo se centra en el diseño de un modelo de casa ecológica con bambú en el área rural de Yantzaza. Los diseños de viviendas hoy en día no están construidos con bambú. Esto se atribuye al desconocimiento de sus ventajas, la desconfianza hacia otras alternativas y la falta de gobierno y de los propios constructores. En el desarrollo de este proyecto, comenzamos con la recopilación de información sobre el bambú, los materiales de construcción alternativos y sus propiedades y cualidades, como su naturaleza económica y su resistencia a los terremotos. Como resultado, recurrimos a recopilar información sobre diversos proyectos, investigaciones y estándares de construcción con bambú de Ecuador, Colombia y Perú. Aquí, se diseñó la parte arquitectónica de la casa, así como sus sistemas activos y la cocina mejorada, y el filtro biológico para el agua potable. El gallinero fue utilizado como cuyera, y el filtro biológico para aguas residuales. En cuanto a la construcción de viviendas, se elaboró un manual para desarrollar su contenido con respecto a los planes que se desarrollaron. Además, se utilizaron revistas, libros electrónicos, manuales y estándares de construcción con bambú. En conclusión, el bambú es un material importante cuyas características principales residen en su comportamiento físico en términos de sus estructuras mecánicas y su relación de peso y resistencia.

Palabras clave: Bambú, Materiales de construcción, Aguas residuales, Filtro biológico 


\title{
Design of a Model of Ecological Housing with Bamboo for the Rural Zone of Yantzaza Zamora Chinchipe, Ecuador
}

\author{
Oswaldo Malla \\ Master en Planificación y Ordenamiento Territorial para el Desarrollo \\ Carlos Hidalgo \\ Magister en Diseño Urbano y Territorial \\ Rita Vásconez \\ Magister en Turismo Sostenible y Desarrollo Local \\ Escuela Superior Politécnica del Chimborazo \\ - Extensión Norte Amazónica, Ecuador
}

\begin{abstract}
The study was developed in the canton Yantzaza, belonging to the province of Zamora Chinchipe. This paper focuses on the design of an ecological house model with bamboo in the rural area of Yantzaza. The designs of houses, nowadays, are not built with bamboo. This is attributed to the ignorance of its advantages, the distrust of other alternatives, the lack of government initiatives, and the builders themselves. In the development of this study, we began with the collection of information about bamboo, alternative construction materials, and their properties and qualities, such as their economic nature and resistance to earthquakes. As a result, we resorted to gathering information on various projects, research, and construction standards with bamboo from Ecuador, Colombia, and Peru. Here, the architectural part of the house was designed, as well as its active systems and the improved kitchen, and the biological filter for drinking water. The chicken coop was used as a guinea pig and the biological filter for wastewater. As for the construction of housing, a manual was developed to improve its content with respect to the plans that were established. In addition, magazines, ebooks, manuals, and building standards with bamboo were used. In conclusion, bamboo is an important material whose main characteristics reside in its physical behavior in terms of its mechanical structures and its relation to its weight and resistance.
\end{abstract}

Keywords: Bamboo, Building materials, Waste water, Biological filter 


\section{Introducción}

El bambú es una planta de gran importancia para los pobladores de las zonas rurales de varias regiones del mundo. Se usa para construcción de casas, antenas de televisión, postes de tendido eléctrico, escaleras, envases, juguetes, muebles, manualidades y para otros usos (Miguel, 2013).

El bambú es un material que tiene importantes características en lo que respecta a su comportamiento físico-mecánico en estructuras. Gracias a su relación resistencia/peso lo catalogan como una de las mejores maderas, con ventajas como lo es la de ser un recurso natural renovable de rápido crecimiento y fácil manejo, que además aporta importantes beneficios ecológicos durante su crecimiento (Jules \& Janssen, 1996). Factores como el desconocimiento del adecuado uso del bambú, intereses de transnacionales y de monopolios de producción de materiales convencionales, las leyes y ordenanzas de construcción locales, la poca apertura de las instituciones viviendísticas y universitarias del país, entre otras causas han contribuido a que sistemas constructivos no convencionales no se hayan masificado, para poder de esta manera solucionar un gran problema social del Ecuador como es el de la vivienda.

Las casas bioclimáticas o ecológicas son aquellas que ofrecen condiciones óptimas de habitabilidad con el mínimo consumo energético, teniendo en cuenta la orientación de la construcción, el terreno y la naturaleza que lo rodea. Deben ser autosuficientes y autorreguladas, con un mantenimiento barato y que no dependan de fuentes no locales de energía (electricidad, gas, carbón o leña). La energía debe proceder mayoritariamente de fuentes naturales gratuitas.

Reutilizando el agua de lluvia se ahorran recursos, y al ser construidas con materiales no tóxicos se integran perfectamente en el medio ambiente, sin perjudicarle. Se deben usar energías limpias y tener cero emisiones. Así mismo, hay que tener en cuenta la integración paisajística de la casa (Cobos, \& León, 2007).

\section{MATERIALES Y MÉTODOS}

El proyecto se desarrolló en la población rural de Yantzaza, en la provincia de Zamora Chinchipe, on un total de aproximadamente 9,476 habitantes. En esta zona existen 2,032 viviendas, de las cuales 1,062 se encuentran en condiciones regulares y 32 en malas condiciones. Dichas viviendas están construidas con material como cubierta de asbesto, zinc, teja, palma, paja Y hoja. (GPE INEN 2, 1976). El presente proyecto tuvo una duración de cinco meses y su objetivo general fue demostrar la sustentabilidad de los recursos naturales, a través del diseño de una vivienda ecológica con bambú para la zona rural de Yantzaza. 
Con base a lo anteriormente descrito se realizó un diseño tridimensional de la vivienda ecológica y de los sistemas activos de la vivienda; ayudándose de los planos base y mediante la aplicación del diseño 3D de AutoCAD 2010.

El diseño y posterior manual de construcción se realizó a partir de la recolección de datos, recopilación bibliográfica para la construcción del conocimiento y la revisión, el análisis y la síntesis de la información científica recolectada en libros, folletos, artículos y proyectistas.

Para determinar los costos de este material se recurrió a fuentes primarias de información como son los propietarios de los aserríos de la localidad. También se buscó información sobre las fuentes de producción, el costo del producto y sus respectivos permisos de aprovechamiento.

Diseño del modelo de vivienda ecológico con bambú para la zona rural del cantón Yantzaza

A través de la utilización de herramientas informáticas como el Software AutoCAD 2010 se realizó un diseño tridimensional de la vivienda ecológica y de los sistemas activos de la vivienda; para esto se partió de los planos base que se diseñaron anteriormente, y se usó la aplicación de diseño 3D de AutoCAD 2010; fueron diseñados los planos base de la vivienda ecológica con bambú tales como los planos arquitectónicos estructurales, eléctricos y sanitarios, donde constan el nombre del proyecto, la ubicación, fecha del diseño, nombre del autor responsable, nombre y versión de la norma utilizada en el diseño y especificaciones de los materiales a utilizarse.

Para el diseño de la vivienda también se tomaron en cuenta las consideraciones cualitativas y cuantitativas del bambú, existentes en las normas y reglamentos de construcciones vigentes de Ecuador como son las normas INEN (Servicio Ecuatoriano de Normalización), normas en las que también constan el tamaño de las ventanas, las puertas, los dormitorios, y la inclinación ideal de una cubierta.

Utilizando el mismo software de diseño se diseñaron, además de la vivienda, los sistemas activos como son: un filtro lento de bioarena, una cocina mejorada, un filtro biológico para aguas residuales, un calentador solar de agua, un pozo de compost, un bihuerto familiar y una granja de gallinas y cuyes. Para poder diseñar estos sistemas se tomaron en cuenta como información base, proyectos ya ejecutados en el país y que fueron adaptados de acuerdo al modus vivendus de la localidad.

Se calculó el costo total de la vivienda, incluidos sus sistemas activos, teniendo en consideración los costos de materiales actuales. Estos montos se obtuvieron en el GAD (Gobierno Autónomo Descentralizado) Cantonal de Yantzaza, y través de estos rubros ya establecidos, se hizo el respectivo cálculo utilizando los planos de la vivienda.

Realización del manual de construcción de la vivienda ecológica con bambú 
Para elaborar el contenido del manual de construcción de la vivienda ecológica se procedió a recopilar información relevante sobre manuales de construcción de viviendas con bambú y de otros documentos de construcción con madera y concreto.

Se describió cada uno de los procesos de construcción de la vivienda, como es la nivelación y replanteo del terreno, la cimentación de muros, las columnas, las paredes, los tipos de uniones, la aplicación correcta del bambú en la estructura de la vivienda y la cubierta. Para desarrollar este contenido se procedió a tomar información del primer objetivo específico, de los planos base y de otros proyectos de construcción conbambú.

\section{RESULTADOS}

Diseño de la vivienda ecológica con bambú

Para la vivienda ecológica con bambú se diseñaron planos arquitectónicos y estructurales donde se reflejan el nombre del proyecto, fecha del diseño, nombre del autor responsable, nombre y versión de la norma utilizada en el diseño, cargas vivas adoptadas, resistencia y especificaciones de los materiales a utilizarse y se aclara que el proyecto es exclusivamente de vivienda.

Planos de la vivienda

Se diseñó una vivienda unifamiliar de un piso para 4 personas, con una altura libre entre pisos de $3 \mathrm{~m}$ y un área total diseñada de $64 \mathrm{~m}^{2}$. Cada área tiene una forma simple y regular sin asimetrías exageradas, las cuales conllevan a concentraciones de fuerzas no deseadas en algunos sectores.

Con base al método planteado y los planos establecidos en el lugar, el diseño de vivienda consistió en lo siguiente:

1) Ejes

Los ejes son líneas punteadas con una numeración consecutiva por un lado y con letras por el otro eje.

Los ejes indicados en el plano coinciden con las líneas que se trazan en el terreno para indicar elementos tales como cimientos, columnas, castillos, muros, etc. Generalmente los ejes están a la mitad de cada elemento estructural, pero en el caso de las colindancias los ejes están en el límite del terreno. 


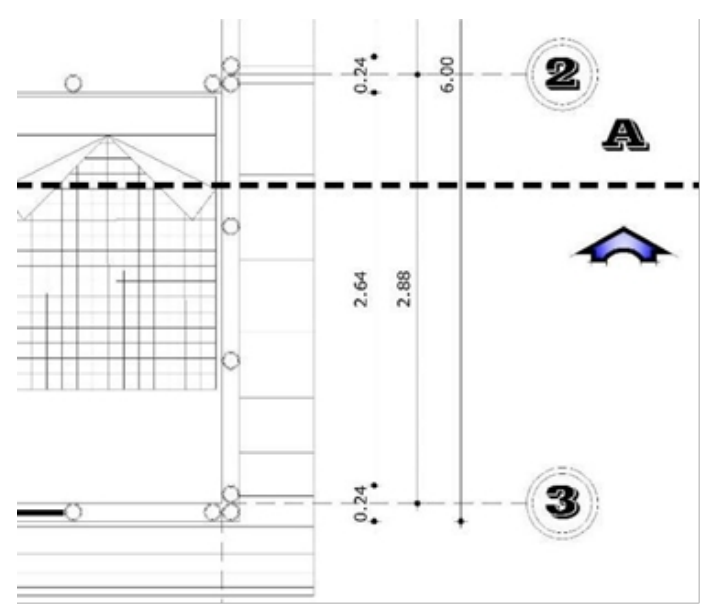

\section{2) Acotaciones}

Las acotaciones son líneas que aparecen cerca de los ejes.

También se conocen a las acotaciones como líneas continuas y se dividen en:

Cotas generales: Proporcionan la distancia total del área a construir.

Cotas parciales: Están en el renglón de abajo de las cotas generales y proporcionan la distancia entre los ejes.

Cotas especificas: Proporcionan las medidas de las puertas, ventanas, etc. 3) Nombre de los espacios

Es el nombre que le corresponde a cada área tales como recámara, sala, cocina, baño, etc. para indicar dónde estarán las instalaciones de agua, luz, gas, teléfono, etc.

4) Representación gráfica

Los muros se representan con líneas gruesas y las ventanas se representan en 2 líneas delgadas y una gruesa.

Las puertas se representan con una línea curva e indica el sentido en que abre o cierra la puerta.

5) Tipos de planos

Plano Arquitectónico: En este plano se muestran los espacios de la vivienda y su distribución; se dibujaron puertas, ventanas, dormitorios, etc.

a) Contenido

Lavandería, Cocina Comedor, Sala, 3 dormitorios, 1 baño, 6 puertas, 1 ventana de ventilación y 2 ventanas de fachada. 


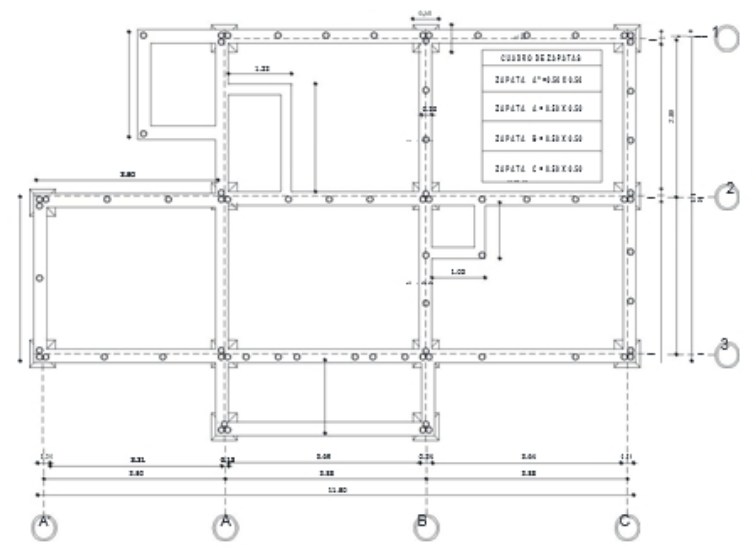

Planos De Estructuras del Proyecto

En toda edificación y en general en toda construcción el primer plano utilizado es el Plano de cimentaciones, que es el que nos indica donde hacer los trazos de las zanjas, su ancho y profundidad, así como las dimensiones de los sobrecimientos donde van ubicadas las columnas, el tipo de concreto a utilizar, etc.

b) Plano de Cimientos

El plano de cimientos generalmente es un plano en planta, dibujado a escala 1:50 en el que se puede observar:

Cimientos: que están limitados por una línea gruesa que debe trazarse sobre el terreno con piola sobre caballetes.

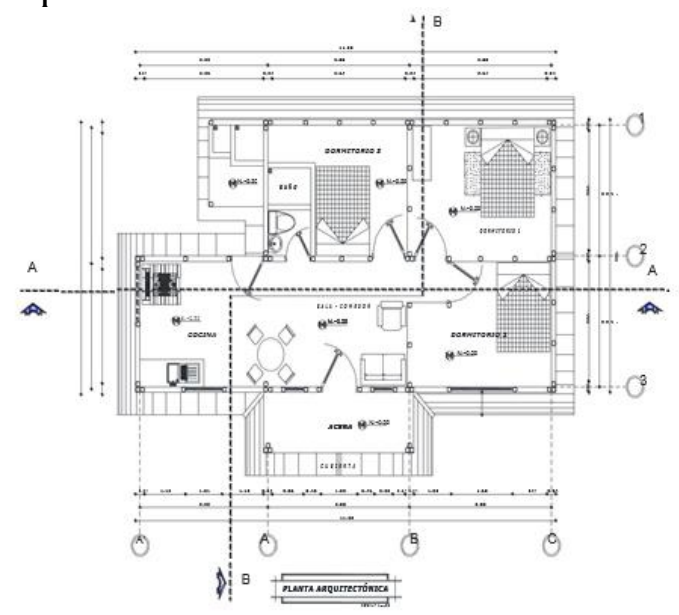

Sobrecimientos: están representados por una línea delgada dibujada dentro de los cimientos indicando el ancho de la pared que soportará. 


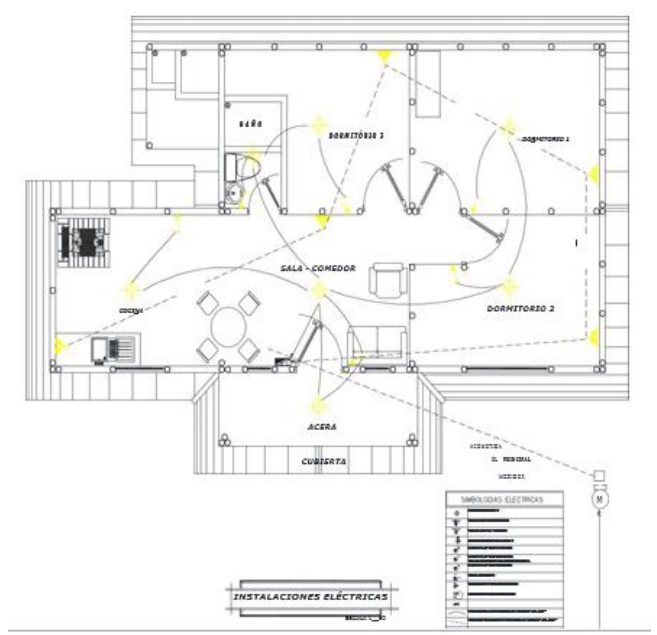

c) Plano Electrónico

Las instalaciones eléctricas de la vivienda requieren del cuidado y del análisis detallado y minucioso de las necesidades para que se garantice que el funcionamiento del sistema sea óptimo y que pueda ser abastecido con suficiente cantidad de energía eléctrica en condiciones de máxima seguridad.

Simbología del dibujo de planos de las instalaciones eléctricas: El cuadro de simbología eléctrica incluye simbología y especificación técnica de los elementos usados.

Instalaciones eléctricas: incluye la acometida eléctrica y red exterior. $\mathrm{La}$ red exterior se refiere a toda aquella instalación especial como iluminación, tomas y otros conceptos que estén incluidos en el diseño.

d) Plano Hidráulico y Sanitario

Las instalaciones sanitarias de la vivienda requieren de cuidado y de un análisis detallado y minucioso para que se garantice que el funcionamiento del sistema sea óptimo y que pueda ser abastecido con suficiente cantidad de agua a presión.

Tubería de aducción: es la tubería que conduce el agua potable desde el sistema del filtro de bio-arena hasta las válvulas de servicio, el tanque cisterna o tanque elevado, según el sistema utilizado.

Simbología del dibujo de planos de las instalaciones Hidráulico y Sanitario

Cuadro de simbología sanitaria que incluye simbología y especificación técnica de los elementos usados. 


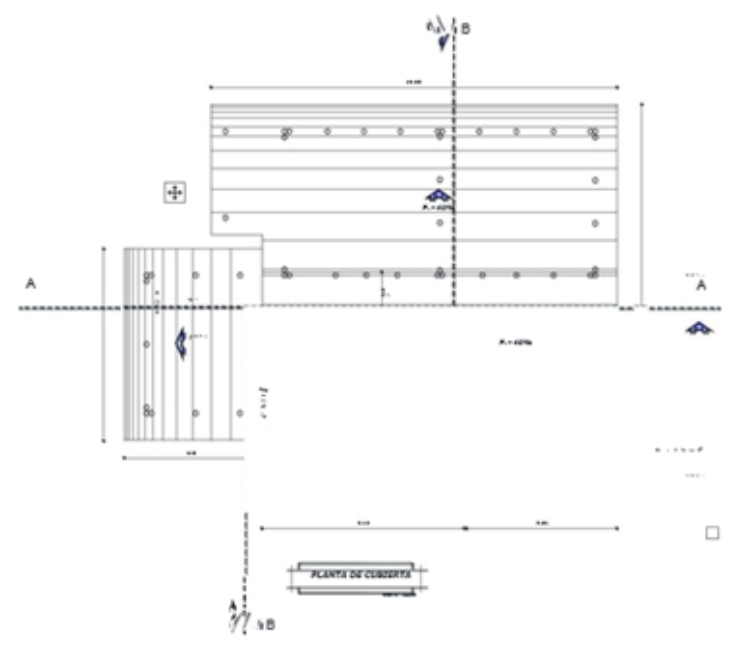

\section{e) Planta de cubierta}

Este plano debe contener lo siguiente:

La estructura de cubierta debe conformar un conjunto uniforme que garantice la estabilidad ante cargas laterales y un correcto soporte y distribución de cargas verticales. Las cubiertas más empleadas en nuestro medio son las de cuatro y las de dos aguas, siendo estas últimas las más útiles en el área urbana.

Para ese tipo de viviendas se recomienda no emplear tejas de barro, las cuales son demasiado pesadas y producen fuerzas mayores que la de tipo liviano al ser aceleradas por el efecto de las ondas sísmicas. En su defecto se deben emplear tejas de asbesto, cemento o similares, las cuales tienen la ventaja de encontrarse en diversos tamaños, colores y texturas, además de que implica la utilización de menos material puesto que permite ampliar las luces entre correas.

Otros de los detalles en la planta son los elementos utilizados en la cubierta, en forma de capas, como el cielo falso, aislante acústico, aislante térmico y la cubierta final. 


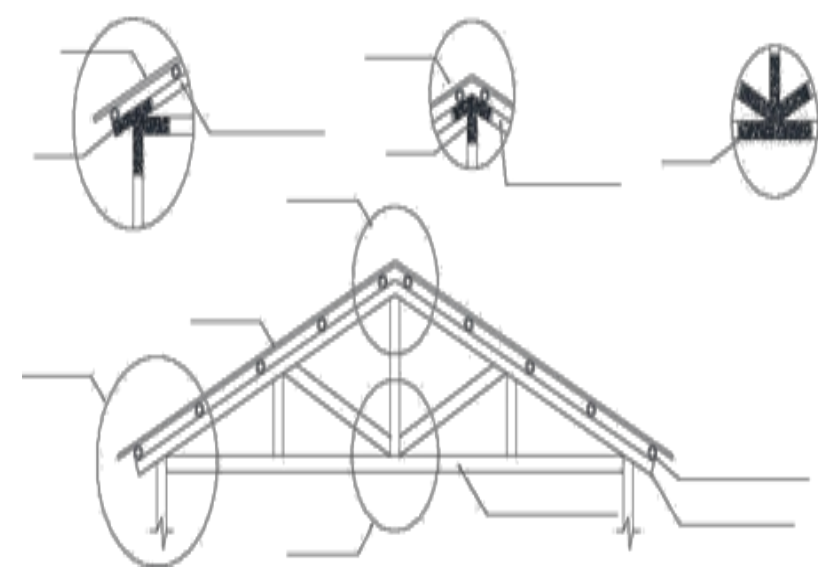

\section{f) Fachadas}

Son planos paralelos a las paredes de la edificación.

En estos planos se deben incluir cotas que indiquen las alturas correspondientes a: vanos de puertas y ventanas, fascias, aleros, batientes, y todo elemento que se encuentre en ellas, acabados etc. El acotado sé hará con relación al nivel de piso terminado \pm 0.00 , que se determinará al momento del diseño de la terracería; generalmente es el nivel interior de piso terminado del edificio en el primer nivel.

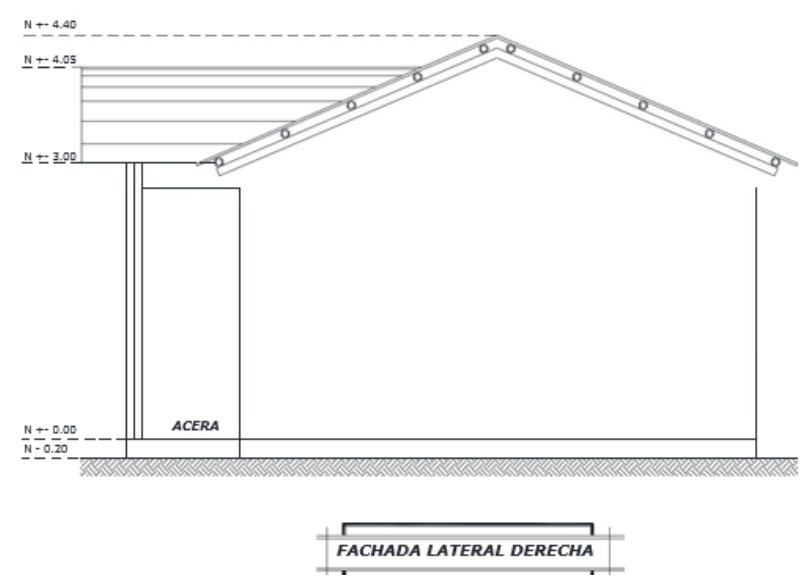



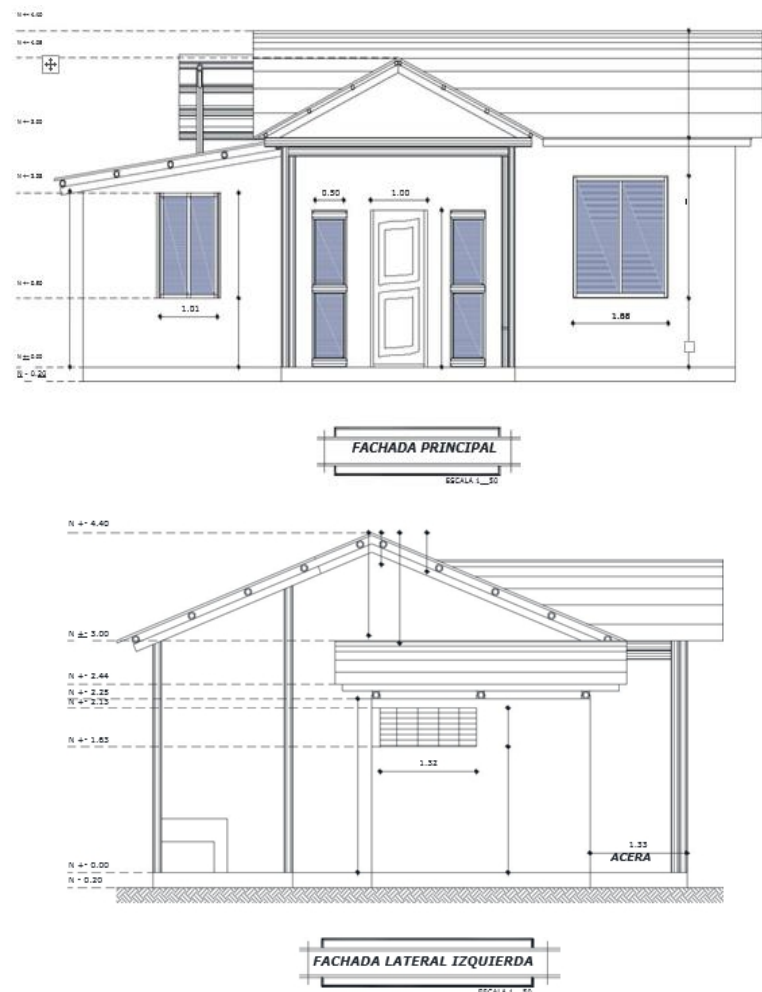

\section{g) Cortes}

Muchos de los detalles de un diseño pueden quedar en el interior y no pueden ser vistos convenientemente en los planos de fachadas y elevaciones o por las vistas en planta. Los planos de corte nos permiten ver mejor los detalles de un objeto que quedan dentro del diseño.

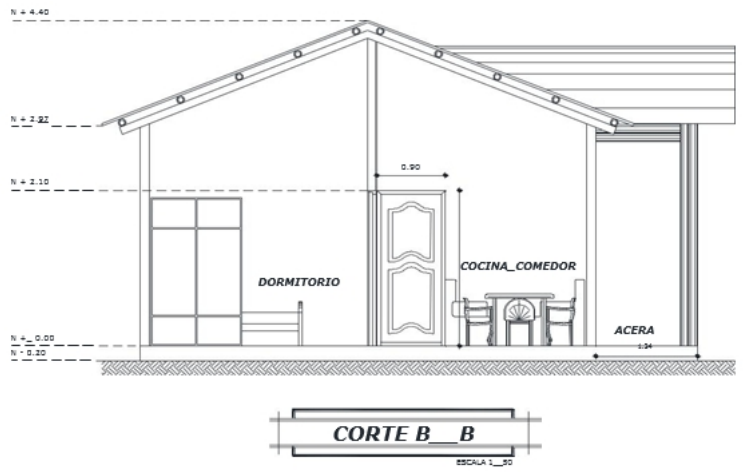

Sistemas activos en el funcionamiento de la vivienda ecológica 


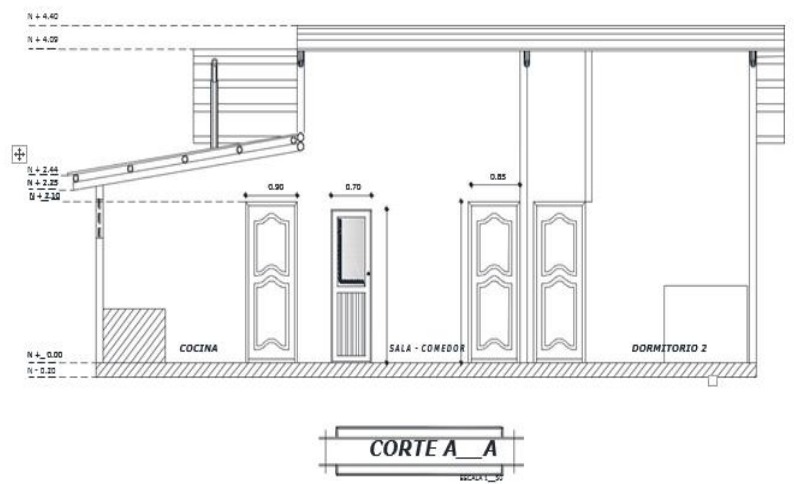

\section{1) Filtro lento de bioarena}

El filtro de bioarena es una adaptación del filtro de arena lento tradicional que permite construirlo a pequeña escala y puede ser operado de manera intermitente. Estas modificaciones hacen que el filtro sea una buena opción para el uso a nivel domiciliario o para pequeños grupos. Puede ser producido localmente en cualquier sitio del mundo porque se construye con materiales fáciles de conseguir.

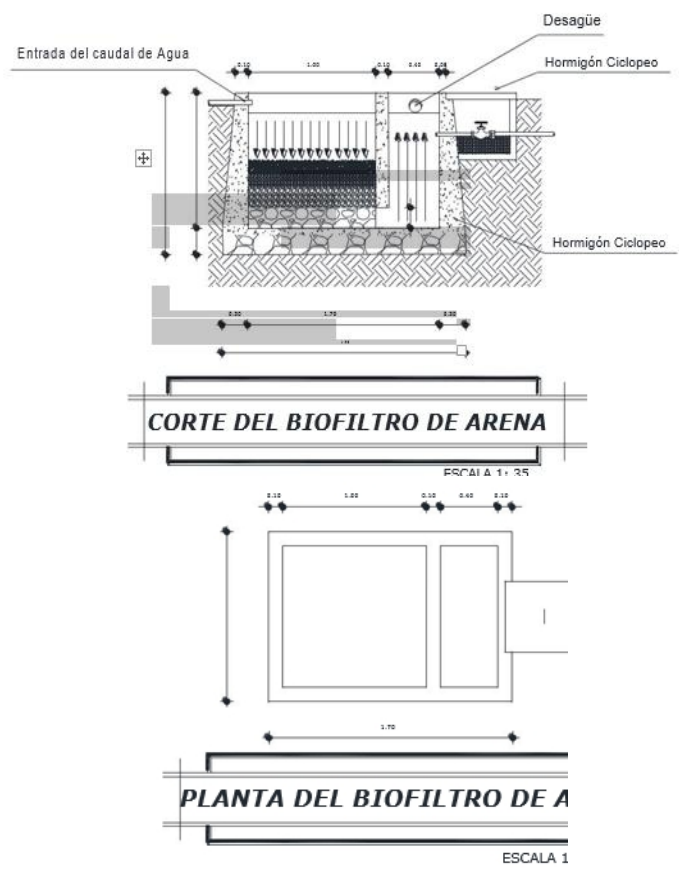

2) Cocina Mejorada

Una cocina mejorada es una cocina que tiene dos hornillas, una cámara de combustión, que es el lugar donde arde la leña, una trampa de ceniza y una chimenea que saca el humo fuera de nuestra cocina. 


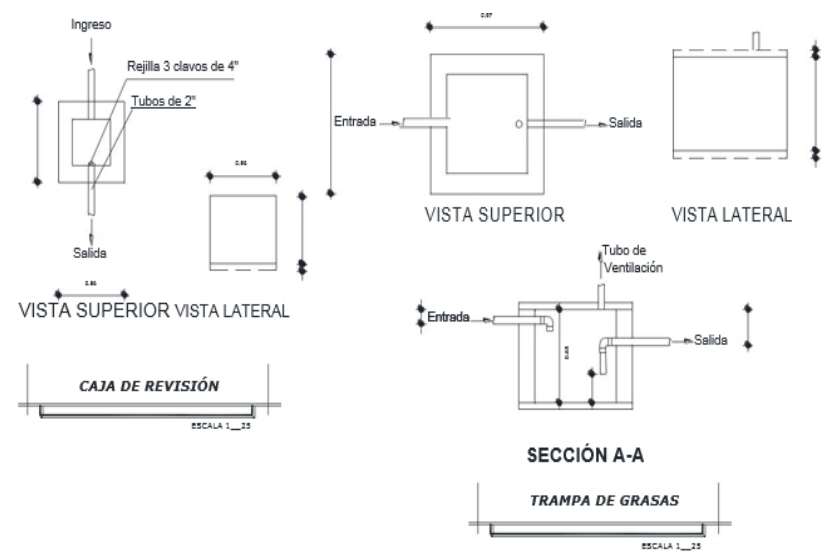

3) Filtro biológico para aguas residuales

El filtro biológico es un sistema destinado a la depuración de las aguas residuales de la vivienda. El proceso consiste en la recolección del agua a través de un sistema de conexión de tuberías al interior de la vivienda, luego pasa por la caja de revisión, una trampa de grasa y finalmente por el filtro de grava, arena y tierra en los cuales a través de un proceso de filtración quedarán todos los residuos atrapados y servirán para que las plantas se alimenten de los nutrientes.
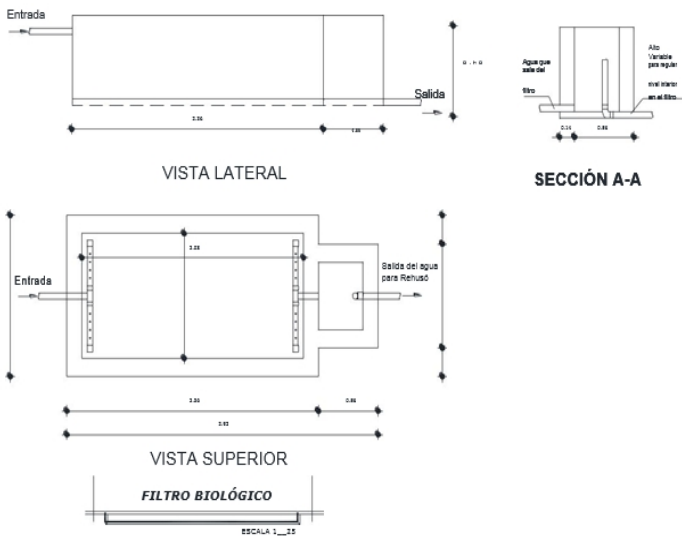

SECCIÓN A-A 

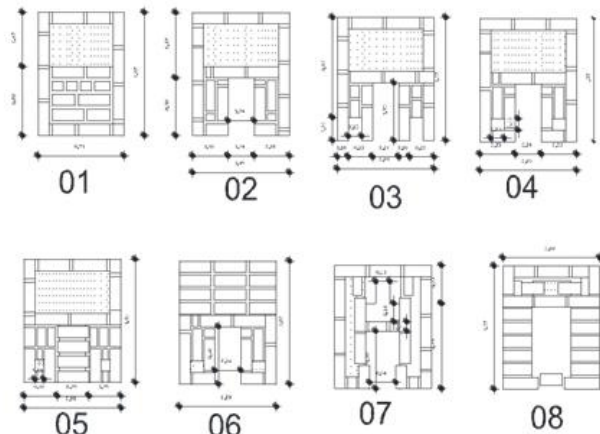

07
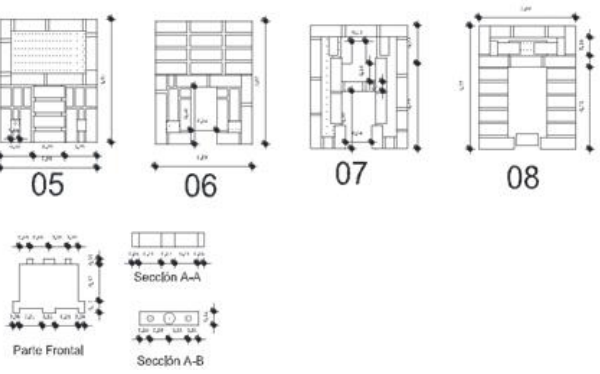

08

\section{4) Terma Solar para Agua Caliente}

Un calentador solar se usa con el fin de aprovechar la energía solar, la cual es gratis y no es aprovechada adecuadamente. Esta energía es limpia, es decir, no produce contaminantes ni daña el medio ambiente como lo hacen los hidrocarburos.
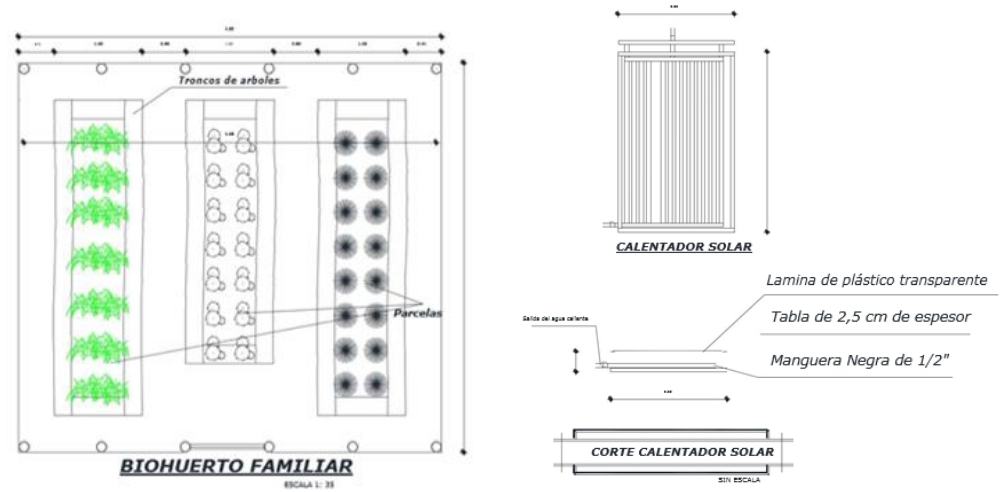

\section{5) Biohuerto familiar}

Busca mejorar la calidad alimentaria de las familias. En especial ayudará a los niños a introducir en su dieta diaria las verduras. Así mismo, se debe fomentar la producción de todo tipo hortalizas en el biohuerto familiar de acuerdo a la zona altitudinal. 


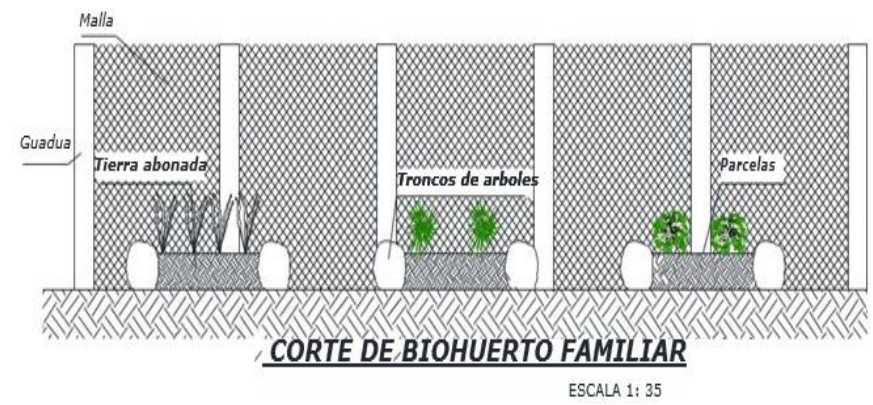

6) Pozo de compost

Las compostas se pueden hacer sobre la superficie del suelo, en pequeños hoyos excavados en el terreno, y pequeños depósitos construidos de cemento, plástico, acero inoxidable y alambre. Los diferentes criterios de elaboración de compostas dependen de las condiciones climáticas. Se recomienda hacer las compostas sobre la superficie del suelo y cuando es menor a esta cantidad, se sugiere que se construyan fosas u hoyos con sistemas de drenaje para recolectar los percolados.

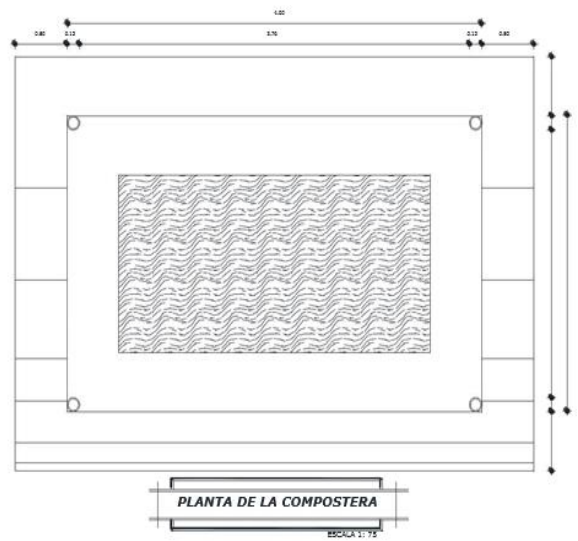

7) Granja de gallinas y cuyes
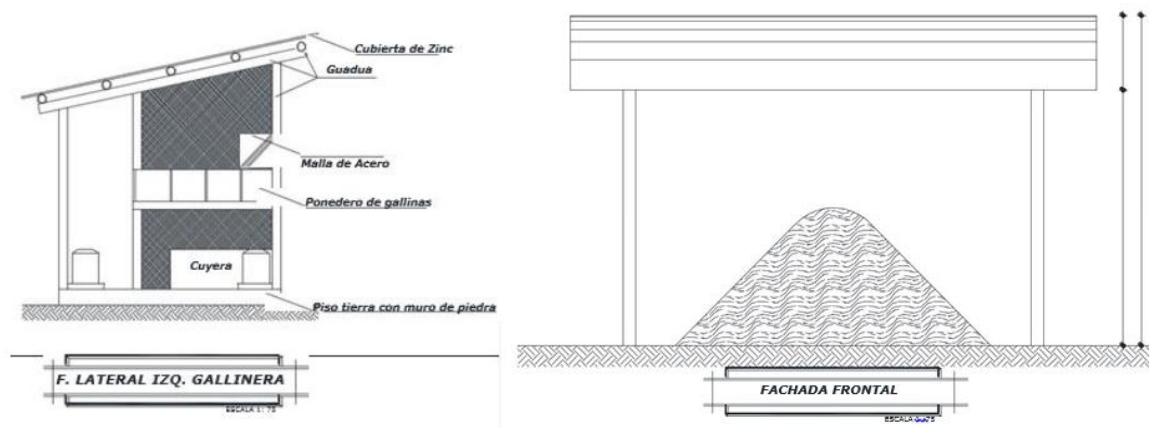
La granja de las gallinas y el cuyero es donde éstos duermen en perchas protegidas de la lluvia, del viento, del frío y libres de depredadores. Aquí también pueden protegerse del sol durante el día y la lluvia.
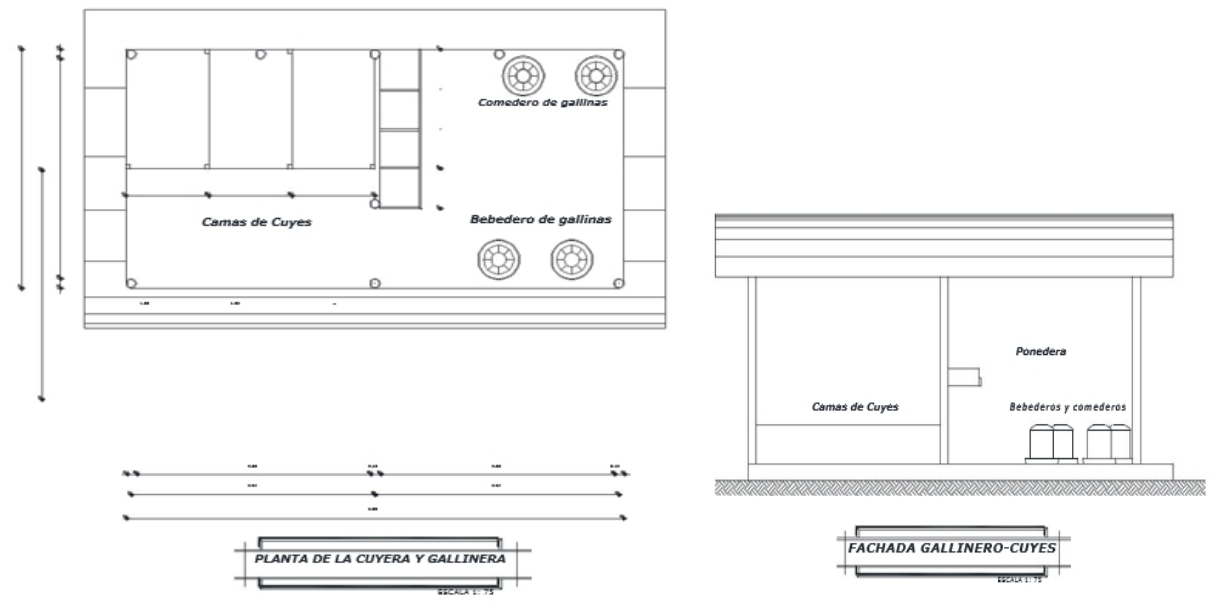

\section{PRESUPUESTO}

El costo de la vivienda ecológica más los sistemas activos que la conforman es \$11869,5 para un período de vida útil de 20 años. Los rubros o costos que se tomaron en cuenta para el cálculo del presupuesto de la vivienda fueron tomados de proyectos que se desarrollan en el cantón Yantzaza, así como también de los proveedores de materiales.

\begin{tabular}{|c|c|}
\hline DESCRIPCION & $\begin{array}{l}\text { COSTO } \\
\text { TOTAL } \\
\end{array}$ \\
\hline TRABAJOS PRELIMINARES & 145,5 \\
\hline CIMENTACIÓN Y ESTRUCTURAS & 1540,7 \\
\hline ACERO Y CUBIERTA & 307 \\
\hline ACEROS EN GENERAL & 613,8 \\
\hline REVESTIMIENTOS & 2505,8 \\
\hline INSTALACIONES ELÉCTRICAS & 403,3 \\
\hline INSTALACIONES SANITARIAS & 256,1 \\
\hline OTROS ELEMENTOS & 3167,9 \\
\hline FILTRO LENTO DE BIOARENA & 554,75 \\
\hline COCINA MEJORADA & 245,83 \\
\hline FILTRO BIOLÓGICO & 454,86 \\
\hline POZO DE COMPOST & 246,24 \\
\hline BIOHUERTO FAMILIAR & 86,63 \\
\hline GRANJA DE GALLINAS Y CUYES & 903,95 \\
\hline CALENTADOR SOLAR & 35,75 \\
\hline TOTAL & 11869,5 \\
\hline
\end{tabular}




\section{DISCUSIÓN}

Información sobre el bambú como un material alternativo para la construcción, sus propiedades, cualidades de bajo costo y resistencia a los sismos.

El bambú se destaca por sus propiedades estructurales entre otras cosas. Su relación resistencia peso excede a la de la mayoría de las maderas, llegando incluso a compararse con el acero y las fibras de alta tecnología. Su capacidad para absorber energía y admitir mayor flexión hace que sea un material ideal para construcciones resistentes a sismos. Sin embargo, este material es susceptible a la humedad y plagas, por lo que se necesitaría realizar una investigación sobre el tipo de plagas que lo afectan y cómo controlarlas.

La Guadua angustifolia, se encuentra en estado silvestre en Colombia, Ecuador y Venezuela, en donde forma colonias dominantes llamadas "guaduales" concentradas principalmente en la región andina, entre los 0 y $2000 \mathrm{msnm}$. No obstante, si analizamos el producto guadua o bambú en Yantzaza, éste es escaso, debido a que ya ha sido explotado para la construcción de andamios en la construcción de viviendas de hormigón, además de las exportaciones a mercados de cantones y provincias vecinas donde la demanda de este producto es alta. Actualmente los principales abastecedores de bambú son provenientes del cantón El Pangui, ya que en este lugar existen bosques primarios a lo largo de las cuencas hídricas, y desde luego el producto es de buena calidad con respecto a la caña guadua que sale de los bosques de Yantzaza.

El bambú se encuentra en estado natural. Su explotación a gran escala disminuye su producción, ya que en la actualidad no existen proyectos para la industrialización del material. La mayoría de los habitantes sólo vende este producto cuando tienen necesidades emergentes y no como un medio para mejorar su economía, es por eso que no se dedican a la producción de bambú en masa.

Diseño de un modelo de vivienda ecológico con bambú para la zona rural del cantón Yantzaza

Para la implementación de la vivienda ecológica con bambú hay que tener en cuenta muchos factores ambientales como la temperatura, la dirección del sol, la humedad, las precipitaciones y la topografía ya que, debido a la fragilidad del material de bambú frente estas condiciones climáticas, se reduce el tiempo de vida útil. Por esto es recomendable entonces el uso de aditivos y presevantes para aumentar su rigidez y resistencia a estos factores.

El ataque de insectos y moho es otro de los aspectos negativos del uso de ese material. En nuestro medio no existen investigaciones sobre cómo contrarrestar este problema. De igual manera, las normas INEN recomiendan este tipo de estudios y según lo investigado en lo que respecta a este material, existen muchos métodos de tratamiento. Es importante resaltar que no se 
puede construir una vivienda con bambú sin pasar por estos procedimientos, ya que esto conllevaría a tener poca durabilidad del material en la construcción de la vivienda ecológica.

También es necesario utilizar brea para recubrir las partes del bambú que se va a cimentar, y el resto de la caña se debe recubrir con pintura de caucho, esto para evitar que le afecte la humedad y los insectos cuando se revistan las paredes. Estas columnas de bambú debenestar recubiertas con mortero y las que permanecen visibles deben pintarse en períodos anuales para conservar sus propiedades mecánicas.

La implementación de los sistemas activos de la vivienda es vital, ya que sin estos no funcionaría el sistema ecológico. ¿Cómo podría denominarse vivienda ecológica si al construirse ésta no tenga un tratamiento adecuado de las aguas residuales que se generan, si los residuos orgánicos que se generan en la vivienda se desechan en el medio ambiente y si no se da un tratamiento adecuado al agua de consumo, lo cual pondría a la familia en riesgo de enfermarse por parásitos? Por ello, estos implementos son importantes ya que mejoran las condiciones de vida de las personas.

El modelo de vivienda ecológica diseñada es solamente para zonas rurales de fácil acceso para el transporte de materiales como el pétreo y de construcción, ya que no se tomaron en cuenta los costos de transporte en acémilas; es decir, el transporte necesario para llegar a lugares de difícil acceso. No obstante, existen alternativas para construir este modelo de vivienda en estas zonas, como por ejemplo no utilizar mortero para revestir paredes, solamente conseguir material para fundir las columnas y muros y utilizar madera en el piso; lo importante de esto es reducir costos energéticos y combinar los materiales al momento de construir una vivienda. En el caso del bambú si existirían inconvenientes de transporte, ya que su adquisición está programada para comprar en los aserríos a un costo de 10 dólares la unidad de $12 \mathrm{~m}$ de longitud, debido a los permisos de explotación exigidos por el Ministerio del Ambiente. Una de las alternativas a largo plazo es que los propietarios de las fincas que no cuenten con este recurso lo cultiven y así aprovechen su potencial constructivo.

\section{Manual de construcción de la vivienda ecológica con bambú}

Este manual brinda procedimientos básicos y describe las buenas prácticas para poder ejecutar con calidad los procedimientos constructivos conbambú. Aunque existen inconvenientes para mostrar información más técnica en los procedimientos constructivos (con lo que se lograría una mejor comprensión por parte del lector), el objetivo de este manual es sólo ofrecer información básica del proceso constructivo, ya que en el campo laboral el profesional que lo ejecute solamente se regirá por los planos de construcción y el presupuesto general de la obra. No obstante, cabe mencionar que la intención ha sido más 
bien ofrecer un medio de apoyo en el caso de que el profesional desconozca cómo hacer uniones o revestidos entre bambú y hormigón.

\section{CONCLUSION}

Tomando en cuenta lo anteriormente expuesto es posible afirmar que el bambú es un material ecológico $100 \%$ sustentable por su rapidez de renovación natural. No es un árbol pero sus tallos son considerados como tales, y está clasificado como una madera semidura a dura. De acuerdo con las más modernas tendencias arquitectónicas, una casa de bambú tiene resistencia, es liviana y prueba que tiene buena capacidad de ahorro de energía.Las construcciones en este material no requieren de alguna herramienta especializada y permiten el uso de mano de obra no tan calificada, aunque debe haber una persona a su dirección con los conocimientos necesarios para su guía; esto contribuye positivamente a la generación de empleo.

De igual manera, el bambú es apto para construcciones sismo-resistentes debido a su rigidez y elasticidad, propiedades que evitan su ruptura al curvarse. Por ejemplo, entre el bambú y el acero el módulo de elasticidad del hormigónbambú es de 150000 hasta $180000 \mathrm{~kg} / \mathrm{cm} 2$, igual al hormigón (acero) de mezcla. Sin embargo, la desventaja está en que el bambú no es conveniente para refuerzo de vigas, por cuanto se producen después muchas grietas en el hormigón. Considerando estas cualidades del bambú, se diseñó una vivienda ecológica, la cual es un sistema sustentable donde interactúan las actividades del hombre en el hogar y la disminución de la contaminación que éste genera, así como el camino hacia el buen vivir, en cuanto a salud, alimentación y la interacción hombre-naturaleza. En el diseño de dicha vivienda ecológica construida con bambú se incluyeron igualmente unos sistemas activos, los cuales son muy importantes para alcanzar la sostenibilidad de un sistema de vivienda ecológico, pues de lo contrario sería un sistema convencional no amigable con el medio ambiente.

\section{References:}

1. Benítez \& Hernández (2002). En elaboración de papel artesanal de caña guadua. Quito, Ecuador: Escuela Politécnica Nacional.

2. Bejarno (2002). Metodología para la construcción de vivienda utilizando como material principal el bambú.

3. Botero (2004). Proyecto: Manual de industrialización del bambú. Buenos Aires, Argentina.

4. Camacho \& Páez (2002). Estudio de Conexiones en guadua solicitada a momento flector. Universidad Nacional de Colombia-Sede Bogotá.

5. Castaño (1990). Algunos sistemas Silviculturales para la propagación y Manejo de la Bambusa guadua en Colombia. Ponencia en el 
Seminario El Bambú: un material de construcción de bajo costo; su cultivo y usos. Santo Domingo, República Dominicana: Enda Caribe.

6. Castro \& Dicken (1985). La guadua: un material versátil. Bogotá, Colombia: Ed. Arco.

7. Castro \& Maza (2013). Proyecto piloto del diseño de una Unidad Educativa Primaria, aplicando tecnologías apropiadas, para el Cantón Puyango. Universidad Internacional del Ecuador.

8. CAWST (2008). Manual Del Filtro Bioarena. Manual CAWST (pág. 5). Obtenido de http://www.cawst.org/es/resources/pubs

9. Cobos \& León (2007). “Propiedades físicas-mecánicas de la guadua angustifolia kunth y aplicación al diseño de baterías sanitarias del IASA II ’. Sangolqui, Ecuador: Escuela Politécnica del Ejército.

10. Colorado (2002). La Guadua, una maravilla de grandes bondades y promisorio futuro. Revista: M\&M, El Mueble y la Madera. No. 34. Árbol De Tinta Ltda.

11. Contreras \& Díaz (s.f.). Inmunización de la guadua. Universidad Nacional de Colombia.

12. CORPEI - CBI (2005). Proyecto CORPEI - CBI "expansión de la oferta exportable del ecuador". Industrialización y Usos del Bambú.

13. CPE INEN 2. (s.f.). Código de práctica para dibujo de arquitectura y construcción. Primera Edición.

14. GPE INEN 2. (1976). Bambú caña guadua. Quito, Ecuador.

15. Cueva \& Maza (2013). "Proyecto piloto del diseño de una unidad educativa primaria, aplicando tecnologías apropiadas, para el cantón Puyango". Loja, Ecuador: Universidad Internacional del Ecuador.

16. Diana (2010). ¿Qué es una casa ecológica? Recuperado el 17 de Febrero de 2014, de Conciencia Eco: http://www.concienciaeco.com

17. Fredd \& Taco (2011). Cálculo y diseño estructural para la cubierta del mercado central de la parroquia de Píntag en base a TensoEstructura con el uso de bambú gigante (Dendrocálamus asper). Escuela Politécnica del Ejército.

18. Giraldo \& Sabogal (1999). Una alternativa Sostenible: La Guadua. Colombia.

19. Gonzalo (2006). La Guadua como material alternativo de la madera. Universidad Tecnológica Equinoccial.

20. Jules \& Janssen (1996). The Bamboo Solution. Recuperado el 17 de Febrero de 2014, de Discovery: http://discovermagazine.com

21. Liese (1985). Bamboos-Biology, silvics, properties, utilization. GTZ. Schotten, Germany: AS-Druck.

22. Lira (2014). Científicos venezolanos estudian el bambú para construir piezas de viviendas a bajo costo. Recuperado el 20 de Febrero de 2014, de Bambú para construcción de viviendas: https:/www.usb.ve/ 
23. Lodoño (2005). La guadua y su aplicación en la construcción. Medellín, Colombia.

24. Lores (2002). Manuales de construcción. Edición, mayo 2012. Lima, Perú.

25. Maldonado (2013). Plan de Ordenación Territorial del Cantón Yantzaza. Yantzaza. Yantzaza, Ecuador.

26. Manual de Diseño para Maderas del Grupo Andino (1984). Proyectos andinos de desarrollo tecnológico en el área de los recursos forestales tropicales. PADT-REFORT. Lima-Perú.

27. Mercado \& Molina (2015). Estudio de factibilidad para la producción de caña Guadua en el recinto de rio chico, cantón Paján de la Provincia de Manabí y propuesta de plan de exportación para el mercado chileno. Universidad Politécnica Salesiana.

28. Mercedes (2006). Guía Técnica Cultivo del Bambú. Etapas de desarrollo de una plantación de bambú. Santo Domingo, República Dominicana.

29. Miguel (2013). Tecnologías para casas y Hoteles Ecológicos (Casas Ecológicas) Tecnologías Apropiadas. Lima, Perú.

30. Moffin (s.f.). Diseño y construcción de un calentador Solar. Proyecto, Desarrollo Tecnológico. Recuperado el 28 de Mayo de 2014, de www.feriadelasciencias.unam.mx

31. Morán (2005). Morán, J. 2005. La Guadua Angustifolia: Antecedentes Históricos, usos tradicionales y actuales posibilidades para el desarrollo social y económico.

32. Nolivos \& Yacelga (2010). Estudio de conexiones entre elementos estructurales de caña guadua sometidos a carga axial. Quito, Ecuador: Escuela Politécnica Nacional.

33. NORMA TÉCNICA. E. 100 BAMBÚ. (s.f.). Recuperado el 24 de Abril de 2014, de www.vivienda.gob.pe

34. Ordoñez, Mejía, \& Barcenas (2002). Manual para la construcción sustentable con bambú. Jalisco, México: Proyecto apoyado por el Fondo Sectorial CONACYT-CONAFOR.

35. Perea, Juan, Yesinith, \& Pilar (2003). Evaluación y documentación de prácticas sobresalientes sobre el manejo de la cosecha y maduración de la guadua en el departamento del Huila. Neiva, Colombia.

36. Rafael (2002). Metodología para la construcción de vivienda utilizando como material principal el bambú. México: Vivienda Modelo CONAFOVI.

37. Shyam \& Nandwani (2006). Calentador Solar de Agua, Construcción, Funcionamiento y Uso. Heredia, Costa Rica.

38. Tandazo \& Flores (2012). "Proceso de industrialización de la caña guadua como material alternativo para la construcción y diseño de 
vivienda tipo de una y dos plantas, empleando caña guadua en sus elementos estructurales". Sangolquí, Ecuador: Escuela Politécnica del Ejército.

39. Telmo \& Flores (2013). Plan de negocio de producción y comercialización de Productos derivado de la caña guadua. Babahoyo, Ecuador: Universidad Técnica de Babahoyo.

40. Terra, F. (2006). Perspectiva Ambiental. Viviendas ecológicas. Barcelona, España.

41. Víctor Mejía \& Guadalupe. (s.f.). Manual para la construcción sustentable con bambú. Métodos de secado. Obtenido de www.conafor.gob.mx 